\title{
Etude Expérimentale et Simulation Numérique de l'Effet des Paramètres Opératoires sur le Processus du Dépôt de Diamant
}

\author{
El Haim, \\ Laboratoire des Sciences Appliquées, Equipe de Modélisation, Optimisation \\ et Dynamique des Structures en Génie Civil, ENSA d'Al Hoceima, \\ Université Abdelmalek Essaadi de Tétouane, Maroc \\ M. Atounti, \\ Laboratoire des Mathématiques Appliquées et des Systèmes d'Information, \\ Faculté Pluridisciplinaires de Nador, \\ Université Mohammed Ir d'Oujda, Maroc

\section{El Hammouti,} \\ Laboratoire Observatoire de la Lagune Marchica de Nador et Région \\ Limitrophe, Equipe des Sciences et de l'Environnement, Faculté \\ Pluridisciplinaires de Nador, Université Mohammed I ${ }^{\text {er }}$ d'Oujda, Maroc
}

Doi:10.19044/esj.2019.v15n30p426 URL:http://dx.doi.org/10.19044/esj.2019.v15n30p426

\section{Résumé}

Ce travail s'intéresse à la croissance des films de diamant par la technique du dépôt chimique en phase vapeur assisté par un plasma microonde (MPACVD) dont la fréquence est $2,45 \mathrm{GHz}$ à partir de mélanges gazeux constitués de $1 \%$ du méthane $\mathrm{CH}_{4}$ contre $99 \%$ d'hydrogène $\mathrm{H}_{2}$. L'ajout de $\mathrm{CH}_{4}$ ne modifie que légèrement la température du mélange, alors cette modélisation numérique focalise l'étude des effets de paramètres essentiels tels que la position du substrat, la pression du mélange gazeux et la puissance incidente sur les caractéristiques physicochimiques d'un plasma micro-onde d'hydrogène pur à basse pression. Le modèle utilisé est un modèle fluide de dérive-diffusion développé sous COMSOL Multiphysics basé sur l'application de la méthode des éléments finis. Cette étude numérique a pour objectif la résolution d'un système d'équations composé de l'équation de continuité, équation de l'énergie des électrons couplées avec l'équation de Poisson. Le comportement physique de la décharge MPACVD, en particulier la densité du plasma, l'uniformité de la décharge ainsi que le volume de la décharge, est simulé et analysé. Les résultats obtenus s'avèrent en accord avec les mesures expérimentales et montrent que ce type de modèle nous permet de mieux comprendre les processus physiques intervenant dans ce type de réacteur à micro-ondes afin d'optimiser ce dispositif. 
Mots Clés: Simulation, densité électronique, modèle fluide, MPACVD

\title{
Experimental Study and Numerical Simulation of the Effect of Operational Parameters on the Diamond Deposition Process
}

\author{
El Haim, \\ Laboratoire des Sciences Appliquées, Equipe de Modélisation, Optimisation \\ et Dynamique des Structures en Génie Civil, ENSA d'Al Hoceima, \\ Université Abdelmalek Essaadi de Tétouane, Maroc

\section{Atounti,} \\ Laboratoire des Mathématiques Appliquées et des Systèmes d'Information, \\ Faculté Pluridisciplinaires de Nador, \\ Université Mohammed Ir d'Oujda, Maroc

\section{El Hammouti,} \\ Laboratoire Observatoire de la Lagune Marchica de Nador et Région \\ Limitrophe, Equipe des Sciences et de l'Environnement, Faculté \\ Pluridisciplinaires de Nador, Université Mohammed I ${ }^{\text {er }}$ d'Oujda, Maroc
}

\begin{abstract}
Microwave plasma assisted chemical vapor deposition (MPACVD) of diamond layers whose frequency is $2,45 \mathrm{GHz}$, have been obtained using a $1 \%$ of methane $\mathrm{CH}_{4}$ with $99 \%$ of hydrogen $\mathrm{H}_{2}$. The addition of $\mathrm{CH}_{4}$ has a slight effect on the $\mathrm{CH}_{4} / \mathrm{H}_{2}$ mixture temperature, so this numerical modeling focuses a study of the effects of the main experimental parameters such as: the substrate position, the gas pressure and the incident power on the physicochemical characteristics of hydrogen microwave plasma at low pressure. This present model is a drift diffusion model developed under COMSOL Multiphysics using the finite element method. The governing equations consist of the continuity equation of electron in drift-diffusion approximation, as well as the energy equation of electron, coupled with the Poisson equation for self-consistent electric field. The physical behavior of the MPACVD discharge, such as plasma density, discharge's uniformity and plasma volume, are simulated and analyzed. The obtained results turn out to be in agreement with previous measurements and show that this kind of model can lead to a better understanding of the physical processes occurring in this kind of microwave reactor and thus allow optimization of this device.
\end{abstract}


Keywords: Simulation, electron density, fluid model, MPACVD

\section{Introduction}

Les dépôts chimiques en phase vapeur assistés par plasma (PACVD) sont de plus en plus utilisés dans l'industrie pour réaliser des revêtements durs sur des surfaces de géométries complexes. Des générateurs de courant continu, pulsé ou non, de radiofréquence ou de micro-onde sont utilisés pour créer la décharge à partir d'un gaz plasmagène.

Dans les réacteurs de dépôt chimique en phase vapeur, les avantages de plasmas micro-ondes sont bien connus. Ils génèrent de fortes concentrations d'espèces chimiques réactives qui peuvent déposer des couches minces à faibles températures du gaz plasmagène, de sorte que les substrats de dépôt ne soient pas endommagés. Par conséquent, les processus fondamentaux mis en jeu au cours d'une opération de dépôt nécessitent une compréhension détaillée des phénomènes de transport liés aux espèces constituant les décharges plasmas micro-ondes dans le but de la conception, la caractérisation et l'optimisation des paramètres de ces décharges.

La mesure des paramètres fondamentaux du plasma (densité et température des électrons) est accessible au moyen de sondes électrostatiques (Swift, D. et al,. 1970). Ce pendant, en condition de dépôt, ces techniques sont difficilement utilisables du fait de l'apparition d'un film sur la partie collectrice des sondes, et aussi du fait que ces sondes jouent le rôle des antennes dans le cas d'utilisation des plasmas micro-ondes (Andrieux, M. et al,. 1998).

En parallèle, la modélisation des décharges plasmas micro-ondes est maintenant considérée comme un outil précieux et très complémentaire à l'analyse expérimentale afin de comprendre la conception et l'optimisation du réacteur à plasma micro-onde pour des applications spécifiques (Lieberman, M. A. et al,. 1994, Rafatov, I. et al., 2012). La simulation numérique d'un tel réacteur, en particulier le réacteur MPACVD, nous permet une mise à l'échelle exacte du réacteur et prédit les paramètres pour un fonctionnement optimal, en ce qui concerne notamment la configuration du réacteur, la composition du gaz, la pression, et les caractéristiques de l'alimentation électrique, etc... (Rafatov, I. et al,. 2012, Gicquel, A. et al,. 1992). La modélisation de ces décharges fournit alors des informations très détaillées sur les systèmes complexes pour lesquels les méthodes analytiques de calcul sont impuissantes. Elle permet ainsi d'accéder à des paramètres physiques du plasma souvent inaccessibles à l'expérience (Hash, D. et al,. 2003). Ceci a été rendu possible par le développement de modèles simplifiés et le choix des techniques de simulation appropriées. Les modèles fluides (El Bojaddaini, M. et al,. 2012, Paraszczak, J. et al,. 1992, Nagayama, K. et al., 1998, Passchir, J. D. P. et al,. 
1993, Kraloua, B. et al,. 2010), particules (PIC-MC) (Bogaerts, A. et al., 2009, Gicquel, A. et al,. 2001), et hybrides (Grotjohn, T. A. et al., 2000, Yamada, H. et al., 2005) sont couramment utilisés pour la simulation numérique des plasmas à basse température (Bogaerts, A. et al,. 2005).

En plus, pendant la dernière décennie, la modélisation des plasmas peut être réalisée dans un temps de simulation qui devient court vu l'augmentation rapide de la performance des systèmes informatiques (El Haim, M. 2015). Les paramètres du plasma dans les réacteurs de dépot chimique en phase vapeur assisté par plasma micro-onde (MPACVD) peuvent être prédis par cette voie avec une grande précision, plus rapidement et à moindre coût (P. Bartos et al, 2008).

Ce travail présente l'apport de la modélisation sur la compréhension des phénomènes intervenant dans la décharge micro-onde de dihydrogène dans les conditions de dépôt du diamant à basse pression. Cette étude a permis de corréler les caractéristiques de cette décharge avec les paramètres de fonctionnement du procédé de dépôt (Andrieux, M. et al., 1998).

\section{Dispositif Expérimental}

Le réacteur à modéliser est un réacteur de dépôt du diament ayant la forme d'un tube cylindrique en quartz de $50 \mathrm{~mm}$ de diamètre interne et de 350 mm de longueur, monté sur deux embouts en acier. Le schéma de principe de ce réacteur est présenté sur la figure.1.

Le tube traverse le guide d'onde rectangulaire dans lequel circule le champ électromagnétique à $2,45 \mathrm{GHz}$ en mode TE (Transverse Electrique) et fourni à partir d'un générateur fonctionnant entre 0 et $2000 \mathrm{~W}$.

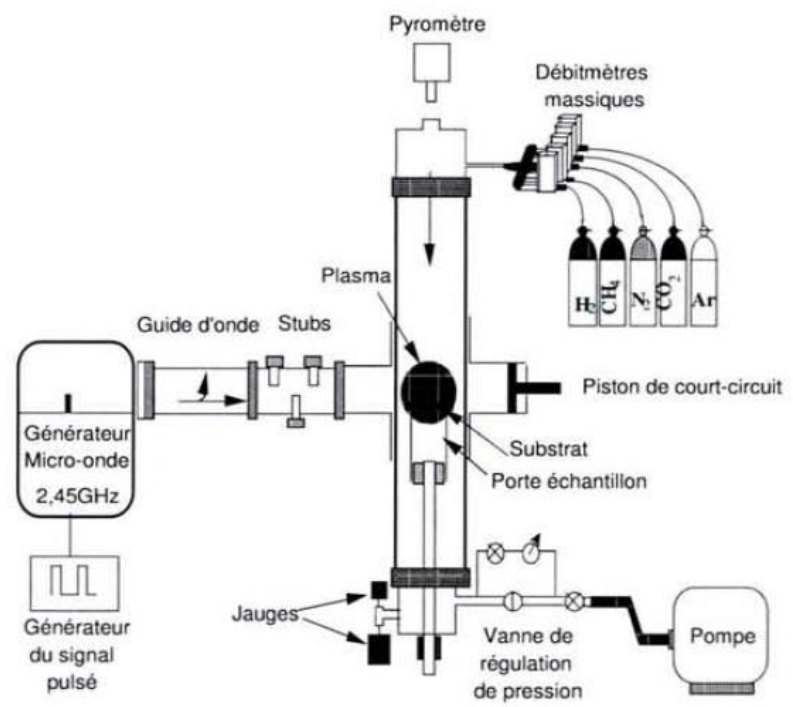

Figure 1. Schéma fonctionnel du réacteur MPACVD de type tubulaire

(Chatei, H. et al., 1997) 
L'onde électromagnétique pénètre à l'intérieur du tube de quartz, puis il va interagir avec le gaz d'hydrogène pour entretenir et créer un plasma microonde au centre de la cavité au niveau de l'intersection du tube et de guide d'onde. Le plasma, limité en volume par le diamètre du tube, s'amorce au centre de la cavité au niveau de l'intersection du tube et du guide, grâce à l'adaptateur d'impédance à vis de réglage (3 stubs) qui lui communique une partie de l'énergie incidente. La puissance réfléchie $(\leq 10 \mathrm{~W})$ est absorbée par un isolateur constitué d'une charge d'eau et un piston de court circuit ce qui permet de créer un système d'onde stationnaire et de régler la position du ventre du champ électrique au centre du tube.

L'activation du plasma se fait par les électrons primaires du gaz plasmagène soumis au champ électrique intense. Une pompe primaire permet d'atteindre un vide limité de $10^{-3}$ Torr et de réguler la pression de travail. La régulation de la pression dans l'enceinte est assurée par une électrovanne située en amont de la pompe. Le porte échantillon est constitué d'un cylindre en quartz dans lequel une cuvette est usinée afin de recevoir l'échantillon. Il recouvre l'extrémité d'une canne de translation en acier inoxydable qui permet d'ajuster la position du substrat dans le plasma (Chatei, H. et al., 1999).

\section{Modélisation Fluide de la Décharge sous COMSOL}

Le modèle utilisé sous COMSOL est un modèle fluide de dérive diffusion ayant pour but de résoudre, dans le domaine spatial et temporel, un système d'équations contenant l'équation de continuité et l'équation de l'énergie des électrons couplées avec l'équation de Poisson (Swift, D. et al., 1970, El Haim, M. et al., 2013).

$>$ Equation de continuité des électrons :

$$
\frac{\partial}{\partial t}\left(n_{e}\right)+\vec{\nabla} \cdot\left[-\left(\mu_{e} \cdot \vec{E}\right) n_{e}-D_{e} \cdot \vec{\nabla} n_{e}\right]=R_{e}
$$

Avec $n_{e}$ est la densité électronique en $\left(1 / \mathrm{m}^{3}\right), R_{e}$ est le terme source de la densité des électrons en $\left(\mathrm{m}^{3} . s\right), \mu_{e}$ est la mobilité des électrons qui est soit un scalaire soit un tenseur en $\left(m^{2} /(V . s)\right), \vec{E}$ est le vecteur champ électrique en $(\mathrm{V} / \mathrm{m}), D_{e}$ est le coefficient de diffusion électronique en $\left(\mathrm{m}^{2} / \mathrm{s}\right)$.

$>$ Equation d'énergie des électrons :

$$
\frac{\partial}{\partial t}\left(n_{\varepsilon}\right)+\vec{\nabla} \cdot\left[-\left(\mu_{\varepsilon} \cdot \vec{E}\right) n_{\varepsilon}-D_{\varepsilon} \cdot \vec{\nabla} n_{\varepsilon}\right]+\vec{E} \cdot \vec{\Gamma}_{e}=R_{\varepsilon}
$$

Avec $n_{\varepsilon}$ est la densité d'énergie des électrons en $\left(V /\left(m^{3} . s\right), \mu_{\varepsilon}\right.$ représente la mobilité d'énergie des électrons en $\left(m^{2} /(V . s)\right), D_{\varepsilon}$ est la diffusivité d'énergie des électrons en $\left(\mathrm{m}^{2} / \mathrm{s}\right)$, et l'indice $\varepsilon$ désigne l'énergie des électrons.

Les coefficients de diffusion électronique $D_{e}$ et la diffusivité d'énergie des électrons sont définis par les relations suivantes: 


$$
D_{e}=\mu_{e} T_{e}, \quad \mu_{\varepsilon}=\left(\frac{5}{3}\right) \mu_{e} \quad, \quad D_{e}=\mu_{\varepsilon} T_{e}
$$

Où $T_{e}$ est la température des électrons qui est une fonction de l'énergie des électrons $\varepsilon$ et elle est définie comme suit:

Où

$$
\begin{aligned}
T_{e} & =\left(\frac{2}{3}\right) \bar{\varepsilon} \\
\bar{\varepsilon} & =\frac{n_{\varepsilon}}{n_{e}}
\end{aligned}
$$

Remarquons alors que si on donne la mobilité des électrons, alors on déterminera les autres paramètres du transport.

$>$ Equation de Poisson:

Pour caractériser une décharge électrique, et en particulier une décharge micro-onde, on doit résoudre les équations de transport représentant le problème, couplées aux équations de Maxwell du fait que le milieu est caractérisé par la présence de particules chargées (éléctrons, ions). Cependant, les équations de Maxwell peuvent se réduire dans ce modèle de dérive diffusion, à l'équation de Poisson définie par:

$$
\vec{E}=-\vec{\nabla} V
$$

$V$ est le potentiel électrique en unité Volt (V).

\section{Géométrie du Réacteur sous COMSOL}

Cette étude s'appuie sur une simulation numérique en 2D basée sur la modélisation fluide sous le logiciel COMSOL Multiphysics. La figure. 2 illustre la géométrie du réacteur à modéliser telle qu'elle a été dessinée sous l'interface de COMSOL.

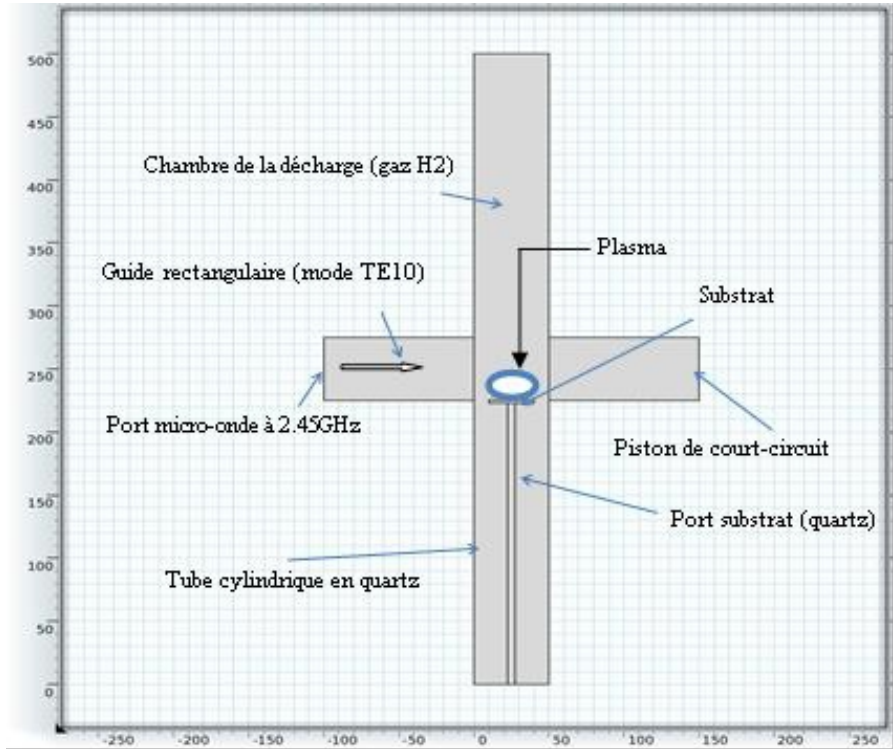

Figure 2.Géométrie du réacteur tubulaire tracée sous COMSOL 
Les conditions aux limites imposées sur les parois du réacteur en quartz, pour la densité des électrons, la température électronique et le potentiel électrique sont comme suit:

$$
n_{e}=0 ; \quad T_{e}=0 \text { et } V=0
$$

La composante tangentielle du champ électrique s'annule sur les frontières en quartz du tube cylindrique. Par conséquent, les ondes électromagnétiques se propageant à ces frontières seraient totalement réfléchies et aucun champ ne pénètre à travers ces surfaces. Cette condition est traduite par la relation suivante:

$$
\vec{n} \times \vec{E}=\overrightarrow{0}
$$

Le port situé à l'extrémité gauche du réacteur est déclaré comme étant le port d'excitation micro-onde où la valeur de la puissance incidente $P_{\text {inc }}$ initiale peut être choisie.

\section{Résultats Numériques et Discussion:}

Ce modèle a pour but de prédire l'effet des paramètres d'entrée, tels que la pression et la puissance incidente ainsi que la position du substrat à l'intérieur du réacteur, sur les caractéristiques du plasma d'hydrogène notamment la densité électronique, l'uniformité du plasma et le volume de la décharge.

L'évolution de la densité moyenne des électrons en fonction de la pression est montrée sur la figure 3.

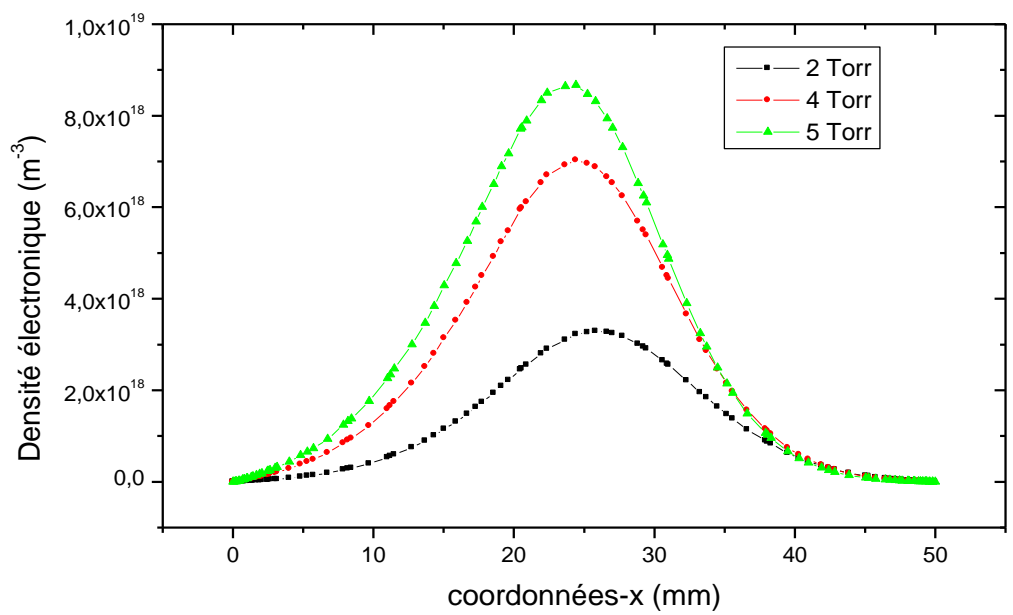

Figure 3. Evolution de la densité électronique en fonction de la position suivant l'axe (o,x) pour différentes pressions et à une puissance incidente de $300 \mathrm{~W}$. 
Il est montré alors que la valeur maximale de la densité des électrons augmente de $3,0.10^{18} \mathrm{~m}^{-3}$ à $8,8.10^{18} \mathrm{~m}^{-3}$, lorsque la pression varie de 2 Torr à 5Torr. Les résultats numériques trouvés, montrent également que la densité des électrons est plus élevée auprès du centre de la décharge $(x=25 \mathrm{~mm})$ et elle diminue pour s'annuler sur les parois du réacteur tubulaire.

Sur la figure. 3 on remarque aussi que, à puissance donnée et au voisinage da la position $\mathrm{x}=40 \mathrm{~mm}$, si la pression est encore augmentée la densité du plasma diminue. Ce comportement est mieux mis en évidence sur la figure.4 représentant une comparaison entre les résultats de simulation et les mesures expérimentales liés à la densité électronique dans une décharge d'hydrogène pur. Ces résultats expérimentaux sont mesurés par la méthode de la double sonde (Chatei, H. et al., 1998). On remarque que les densités électroniques moyennes, calculées et mesurées, diminuent en fonction de la pression du gaz, et que la densité moyenne des électrons reste dans la gamme de l'orde de grandeur $\left(10^{17}-10^{18}\right) m^{-3}$, pour une puissance incidente constante (300W).

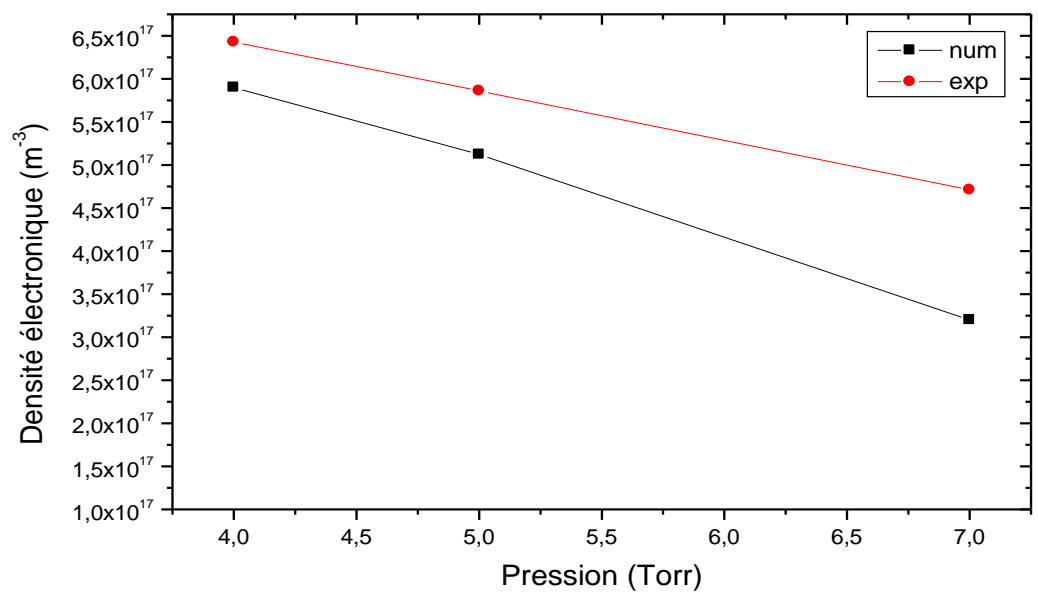

Figure 4. Evolution de la densité électronique en fonction de la pression à une puissance incidente de $300 \mathrm{~W}$ et pour la position $\mathrm{x}=40.3124939 \mathrm{~mm}$

Jusqu'à 4Torr, l'augmentation de la densité avec la pression est due à l'amélioration de l'efficacité du transfert d'énergie collisionnel du champ aux électrons. En effet, la puissance moyenne absorbée par un électron $\mathrm{P}_{\mathrm{abs}}$ augmente avec la fréquence de collisions $v$, et donc avec la pression, tant que le rapport $v / \omega$ est inférieur à 1 , ce qui est toujours le cas dans notre domaine de pressions. Au-delà de 4Torr, bien que le rapport $v / \omega$ soit toujours inférieur à 1 , la décroissance de la densité du plasma observée s'explique par une réduction de la longueur de diffusion du plasma lorsque la pression augmente. 
Ce phénomène est accentué par le désaccord d'impédance résultant de l'augmentation de la pression (Bartos, P. et al., 2008).

Dans une autre étude, en augmentant la puissance incidente et et en maintenant la pression du gaz plasmagène constante, on peut obtenir les profils de la densité électronique comme présentés sur la figure 5.

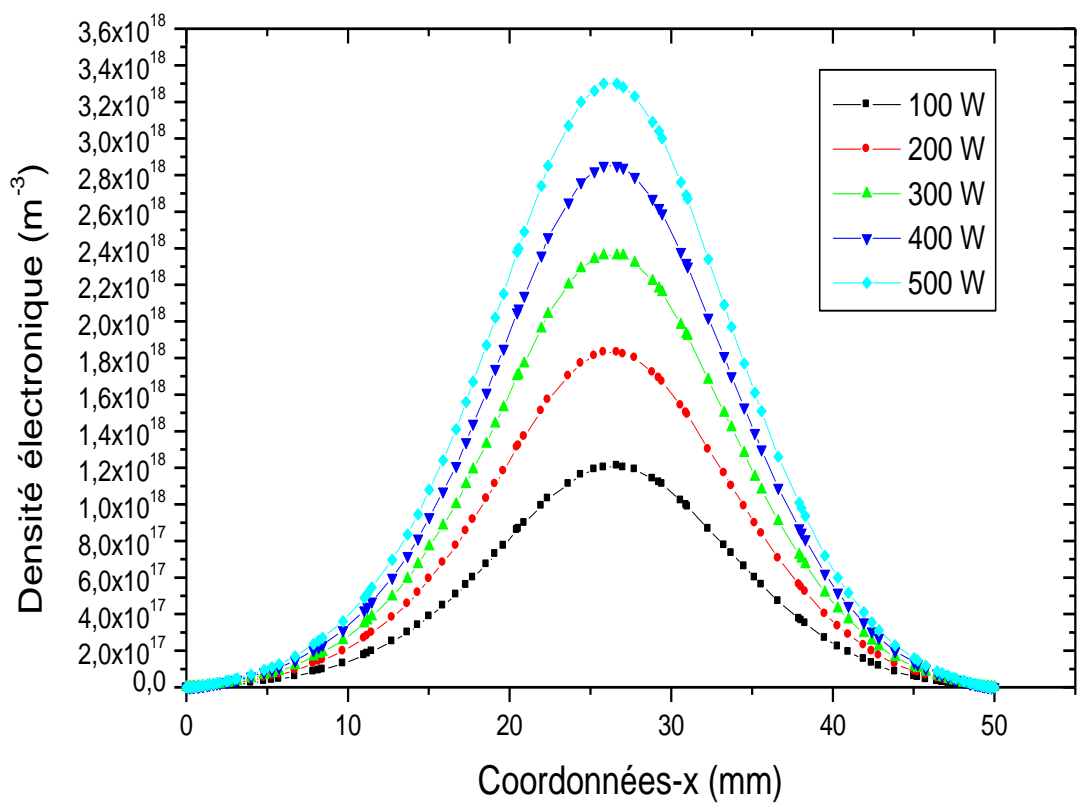

Figure 5. Evolution de la densité électronique en fonction de la position suivant l'axe (o,x) pour différentes puissances et à une pression de 1.5 Torr

Il est clair que la densité électronique atteint sa valeur maximale au centre de la décharge où $\mathrm{x}=25 \mathrm{~mm}$, et elle diminue lorsqu'on s'éloigne vers les bords du plasma. Et en plus, la valeur maximale de la densité électronique augmente de $1,2.10^{18} \mathrm{~m}^{-3}$ à $3,3.10^{18} \mathrm{~m}^{-3}$ lorsque la densité de puissance varie de $100 \mathrm{~W}$ à $500 \mathrm{~W}$ (El Haim, M. et al., 2013).

On rappel que l'obtention de plasmas denses (de l'ordre de $1,0.10^{18} \mathrm{~m}^{-3}$ ) et uniformes sur le substrat de dépôt des couches minces du diamant s'inscrit parmi les objectifs de ce travail. A ce stade, selon les résultats présentés cidessus, cet objectif est atteint.

Du point de vue de la maîtrise des procédés de dépôt, il est impératif de chercher la position adéquate du substrat de dépôt et aussi bien les potentiels maximums qui peuvent provoquer des décharges denses au voisinage du centre du substrat. Ce paramètre est présenté dans la figure 7. 


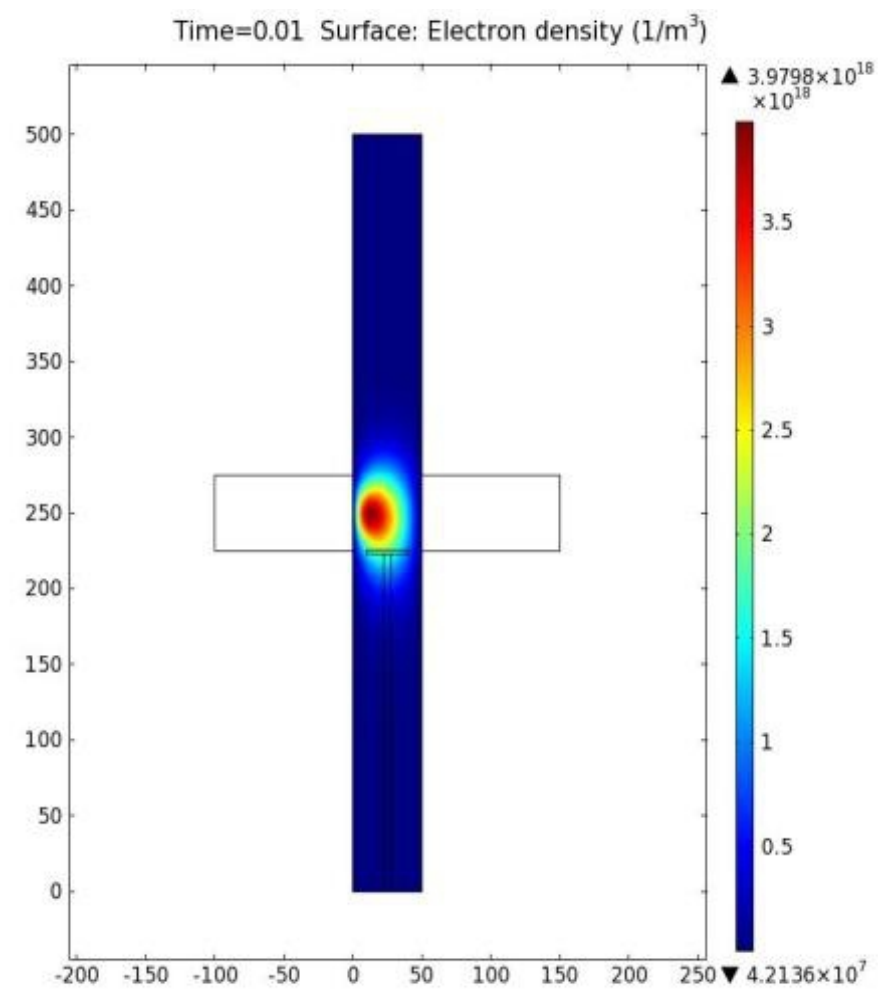

Figure 6. Profil de la densité électroniques imulé à $300 \mathrm{~W}$ et à 1,5Torr après: $0,01 \mathrm{~s}$

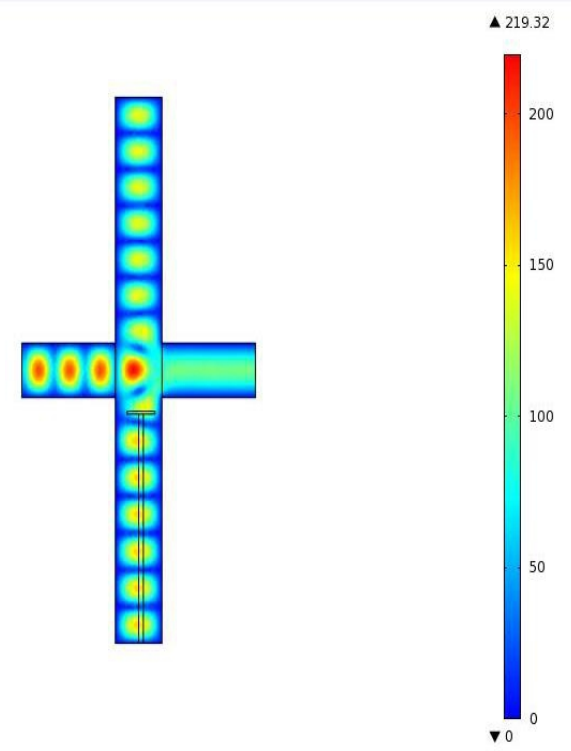

(a) Position du sbstrat: $y=210$ $\mathrm{mm}$

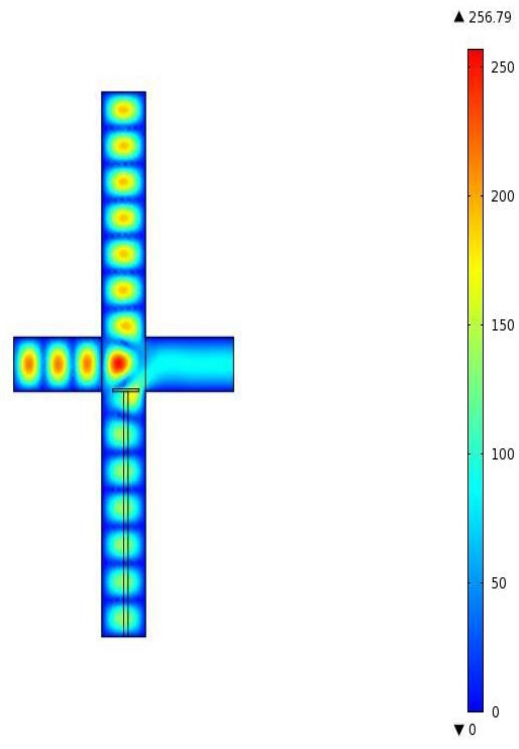

(b) Position du sbstrat: $y=$ $225 \mathrm{~mm}$ 

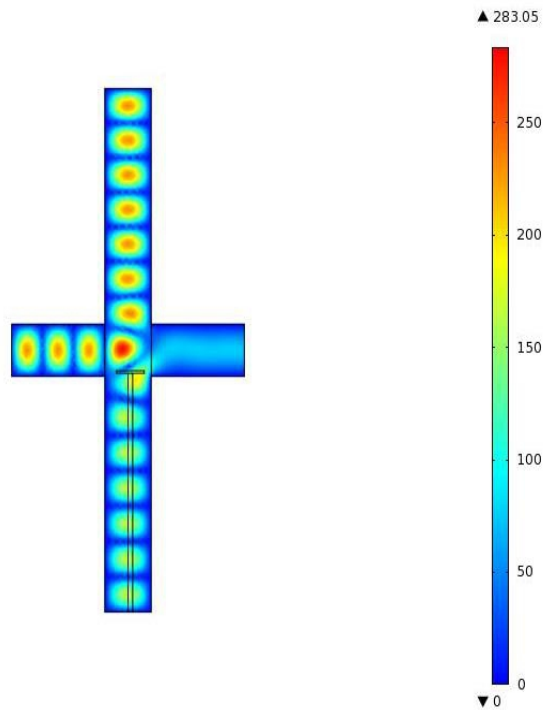

(c) Position du sbstrat: $y=228 \mathrm{~mm}$

Figure 7. Uniformité du plasma pour différentes positions du substrat

Le profil de la décharge trouvée dans les conditions où le substrat est situé à y $=228 \mathrm{~mm}$ est montré dans la figure 6 . On en déduit alors l'impact de la position du substrat de dépot sur l'uniformité et le volume de la décharge.

\section{Conclusion}

Cette étude est consacré à la modélisation fluide d'un plasma microonde d'hydrogène pur créé et entretenu dans un réacteur tubulaire sous les conditions de dépôt de diamant à basse pression. Le modèle fluide appliqué repose sur le couplage des équations de continuité des électrons, de l'équation de l'énergie pour les électrons et de l'équation de Poisson. En outre, à l'aide du logiciel COMSOL Multiphysics on a pu approximer la solution de ce système d'équations en appliquant la méthode des éléments finis. Des résultats numériques satisfaisants ont été obtenus dans cette étude et des comparaisons avec les résultats expérimentaux ont montré un bon accord. En effet, on a pu mettre en évidence l'impact des principaux paramètres physiques tels que la pression, la puissance micro-onde incidente et la position du substrat sur les caractéristiques fondamentales de la décharge à savoir essentiellement la densité électronique et l'uniformité ainsi que le volume du plasma. Le bon accord des résultats numériques trouvés avec les résultats expérimentaux, où la densité électronique est de l'ordre de $10^{18} \mathrm{~m}^{-3}$, nous permet d'optimiser une conception du réacteur et de ses conditions de fonctionnement. Ceci, avant 
d'ajouter le gaz du méthane $\mathrm{CH}_{4}$ pour élaborer des films de diamant dans des conditions optimales.

\section{References :}

1. Andrieux, M., Badie, J. M., Ducarroir, M., Thomas, L. (1998). Etude par Spectroscopie d'Emission Optique de Décharges Radiofréquence et Micro-Onde Lors du Dépôt Chimique en Phase Vapeur (PACVD) dans le Système Si-C-Ar. Emission Optique de Décharges en PACVD, Ann. Chim. Sc. Mat, No 23, pp. 743-752.

2. Bartos, P., Hrach, R., Jelinek, P. (2008). Multidimensional FluidParticle Modeling Technique in Low-Temperature Argon Plasma at Low Pressure, Science Direct, Vacuum 82, pp. 220-223.

3. Bogaerts, A., Bultinck, E., Eckert, M., Georgieva, V., Mao, M., Neyts, E., Schwaederle, L. (2009). Computer Modeling of Plasmas and Plasma-Surface Interactions. Plasma Process. Polym. N6, pp.295307.

4. Chatei, H., Bougdira, J., Remy M., Alnot, P. (1999). Optical Emission Diagnostics of Permanent and Pulsed Microwave Discharges in H2CH4-N2 for Diamond Deposition, Surf. And Coat. Technol. Vol. 116119, pp. 1233-1237.

5. Chatei, H., Bougdira, J., Remy M., Alnot, P. (1998). Mechanisms of diamond films deposition from MPACVD in methane-hydrogen and nitrogen mixtures, Surface and Coatings Technology 98, pp. 10131019.

6. Chatei, H. (1997). Etude du Procédé de Croissance du Diamant en Plasma Micro-Onde. Thèse de Doctorat d'Etat, Faculté des Sciences, Université Mohamed Ier - Oujda, Maroc.

7. El Bojaddaini, M. (2014). Modélisation fluide d'un plasma micro-onde dans les conditions de dépôt de diamant. Thèse de Doctorat National, Faculté des Sciences, Université Mohamed Ier - Oujda, Maroc.

8. El Bojaddaini, M., Chatei, H., Atounti, M., El Haim, M., Driouch, I., El Hammouti, M.(2012). Numerical Simulation of Hydrogen Microwave Plasma Discharge Using a Fluid Model Approach, Applied Mathematical Sciences, Vol. 6, N. 121, pp. 6003-6019.

9. El Haim, M., El Hammouti, M., Chatei, H., Atounti, M., El Bojaddaini, M. (2013). COMSOL Multiphysics Simulations of the Hydrogen Microwave Plasma Characteristics. International Review on modelling and Simulations (I.RE.MO.S), Vol. 6, No. 6, pp. 1979-1986.

10. El Haim, M. (2015), Modélisation Numérique et Caractérisation des Décharges Micro-ondes : Application aux Réacteurs MPACVD. Thèse de Doctorat National, Faculté des Sciences, Université Mohammed Ier - Oujda, Maroc. 
11. Gicquel, A., Hassouni, K., Silva, F. Achard, J. (2001). CVD Diamond Films: From Growth to Applications. Current Applied Physics. $\mathrm{N}^{\circ} 1$, pp. 479-496.

12. Grotjohn, T. A., Asmussen, J., Sivagnaname. J., Story, D., Vikharev, A. L., Gorbachev, A., Kolysko, A. (2000). Electron Density in moderate Pressure Diamond Deposition Discharges. Diamond and Related Materials, $\mathrm{N}^{\circ}$ 9, pp. 322-327.

13. Haagelaar, G. J. M., Pitchford, L. C. (2005). Solving the Boltzmann Equation to Obtain Electron Transport Coefficients and Rate Coefficients for Fluid Models. Plasma sources Sci. Technol., No 14, pp. 722-733.

14. Lieberman, M. A., Lichtenberg, A. J. (1994). Principles of Plasma Discharges and Materials Processing, John Wiley, New York.

15. Kraloua, B., Hennad, A.(2010). Bidimensional Modelling NonEquilibrium Fluid Model of Glow Discharge at Low Pressure. International Review on Modelling and Simulations (I.RE.MO.S), Vol. 5, N.6, pp. 2653-2656.

16. Nagayama, K., Farouk, B., Lee, Y. H. (1998). Particle simulation of radio-frequency plasma discharges of methane for carbon film deposition. IEEE Transactions on Plasma Science, 26, 125.

17. Paraszczak, J., Heidenreich, J. (1992). Applications of microwave plasmas in microcircuit fabrication, chapter 15. Microwave Excited Plasmas, edited by Michel Moisan, Jacques Pelletier, (Plasma Technology, 4).

18. Passchier, J. D. P., Goedheer, W. J. (1993). A Two-Dimensional Fluid Model for an Argon RF Discharge. J. Appl. Phys. Vol. 74 N6. Pp. 37-44.

19. Rafatov, I., Bogdanov, E. A., Kudryavtsev, A. A. (2012). On the Accuracy and Reliability of Different Fluid Models of the Direct Current Glow Discharge, Phys. Plasmas 19, (V. 033502).

20. Swift, D., Shwar, M. J. R. (1970). Electric Probe for Plasma Diagnostic, London ILIFFE Books LTD.

21. Yamada, H., Chayahara, A., Mokuno, Y., Soda, Y., Horino, Y., Fujimori, N. (2005). Modeling and Numerical Analyses of Microwave Plasmas for Optimizations of a Reactor Design and its Operating Conditions. Diamond \& Related Materials, Vol. 14, Issues. 11-12, pp. 1776-1779. 